\title{
Geometric and electronic structure of lanthanide orthophosphate nanoparticles determined with $\mathrm{X}$-rays
}

\author{
E. Suljoti, ${ }^{1, a), b)}$ M. Nagasono, ${ }^{1, c)}$ A. Pietzsch, ${ }^{1}$ K. Hickmann, ${ }^{2}$ D. M. Trots, ${ }^{3}$ M. Haase, ${ }^{2}$ \\ W. Wurth, ${ }^{1}$ and A. Föhlisch ${ }^{1, a), d)}$ \\ ${ }^{1}$ Institut für Experimentalphysik, Universität Hamburg, Luruper Chaussee 149, \\ D-22761 Hamburg, Germany \\ ${ }^{2}$ Institut für Chemie, Universität Osnabrück, Barbarastrasse 7, D-49067 Osnabrück, Germany \\ ${ }^{3}$ HASYLAB at DESY, Notkestraße 85, D-22607 Hamburg, Germany
}

(Received 26 July 2007; accepted 23 January 2008; published online 3 April 2008)

\begin{abstract}
The evolution of the geometric and electronic structures within the entire series of lanthanide orthophosphate nanoparticles $(\sim 2-\sim 5 \mathrm{~nm})$ has been determined experimentally with X-ray diffraction and near edge X-ray absorption fine structure spectroscopy. In particular, the interplay between electronic structure, crystal morphology, and crystal phase has been systematically studied. A missing local order in the crystal structure accompanied by multiple ion sites in the nanoparticles was revealed to be due to the small crystal size and large surface contribution. All lanthanide ions were found to be in " $3+$ " configuration and accommodated in three different crystallization states: the larger lanthanide ions ( $\mathrm{La}, \mathrm{Ce}, \mathrm{Pr}, \mathrm{Nd}, \mathrm{Sm})$ in the monoclinic phase, the smaller ones $(\mathrm{Er}, \mathrm{Tm}$, $\mathrm{Yb}, \mathrm{Lu})$ in the tetragonal phase, and the intermediate lanthanide ions ( $\mathrm{Eu}, \mathrm{Gd}, \mathrm{Tb}, \mathrm{Dy}, \mathrm{Ho}$ ) in a "mixed phase" between monoclinic and tetragonal phases. () 2008 American Institute of Physics. [DOI: $10.1063 / 1.2876360]$
\end{abstract}

\section{INTRODUCTION}

Nanoscience holds the promise to tailor the physical, chemical, and biological properties of matter, ${ }^{1}$ evolving from "infinitely extended" solids toward nanometer-scale solids consisting of a defined cluster of atoms. In semiconductors, this evolution in size is accompanied by quantum confinement of mobile charge carriers. As a consequence, the band gap, optical properties, melting temperature, and radiative lifetime of the lowest allowed optical transition can be varied in semiconductors as a function of crystal size. ${ }^{2}$ In wide band gap insulators, size related changes lead to changes in the electronic and geometric structures, i.e., the oxidation state, the crystal phase, or unit cell distortions. Lanthanide phosphate nanoparticles are an interesting class of wide band gap insulators because of their optical properties. Their colloidal solutions emit infrared, visible, and ultraviolet $\operatorname{light}^{3-7}$ and, therefore, they can be used as components in lighting, displays, optoelectronic devices, lasers, and in long-lived dyes for biological labeling. ${ }^{5}$ The lanthanide orthophosphates also display very high lattice energies and high resistance against oxidation and radiation damages, making bulk crystals of these materials a medium feasible even for nuclear-waste storage. ${ }^{8}$ Thus, it is essential to understand how dimensionality influences the properties of lanthanide orthophosphates. Recent studies ${ }^{9,7,10,6}$ have provided some insights on how crystal structure and kinetic factors determine the shape and size of these materials. However, an important issue to be

\footnotetext{
a) Authors to whom correspondence should be addressed.

${ }^{b)}$ Electronic mail: edlira.suljoti@desy.de.

${ }^{c}$ Present address: RIKEN/XFEL Project Head Office, Kouto 1-1-1, Sayo, Hyogo 679-5178, Japan.

${ }^{\mathrm{d})}$ Electronic mail: alexander.foehlisch@desy.de.
}

addressed is the question of how morphology and geometric structure influence the electronic structure of these materials. Most of the reported studies are focused on their optical properties and have revealed the luminescence quantum efficiency to be very small compare to bulk materials. The intrinsic emission of pure $\mathrm{LaPO}_{4}$ nanoparticles was reported to be ten times smaller in intensity than the microsized particles. ${ }^{11}$ The luminescence quantum yield efficiencies of $\mathrm{CePO}_{4}: \mathrm{Tb}$ and $\mathrm{LaPO}_{4}:$ Eu colloidal solutions were reported to be $16 \%$ and $10 \%$, respectively, ${ }^{3}$ and for the $\mathrm{LaPO}_{4}$ doped with different lanthanides, $\mathrm{Pr}, \mathrm{Nd}, \mathrm{Er}$, and $\mathrm{Yb}(3+)$ ions was reported to be less than $15 \% .^{5}$

The measured decay curves of the optical transitions in the nanoparticles showed shorter mean lifetimes (range from 1.6 up to $2.3 \mathrm{~ms}$ ) compared to their bulk crystals. ${ }^{12,5} \mathrm{An}$ additional reported aspect is the shift toward lower energies of the excitonic emission band in pure $\mathrm{LaPO}_{4}$ nanoparticles ${ }^{11}$ and of the $5 d-4 f$ transition band in $\mathrm{CePO} 4$ nanoparticles ${ }^{13}$ compared to their bulk crystals. Besides, the intensity and the splitting of Eu optical transitions was shown to depend on the morphology and the crystal structure of the host lattice. ${ }^{6}$ All these aspects of the optical properties address for a systematic and detailed study of the electronic structure of the whole series of lanthanide orthophosphate nanoparticles. As a structural probe we have used the variation of the oxygenlanthanide chemical bond length because it can be probed for the entire lanthanide orthophosphate series.

The nanoparticles of lanthanide orthophosphate series show to adopt different crystal phases and different morphologies depending on the synthetic method being used. The reason for this is that bulk lanthanide orthophosphates have several polymorphic forms; hexagonal, tetragonal, and monoclinic phases. Yan et al. ${ }^{6}$ have reported the fabrication 
of the whole orthophosphate series in the nanosize ranges based on the same hydrothermal method. In the case of larger lanthanide ions ( $\mathrm{La}, \mathrm{Ce}$, and $\mathrm{Nd}$ ), they reported the orthophosphates to adopt the monoclinic structure and rodlike morphologies with widths of 20-30 nm and lengths up to $1 \mu \mathrm{m}$. The lanthanide ions of intermediate size (Pr-Tb) preferred a partly hydrated hexagonal phase and highly anisotropic rodlike morphologies with widths of $10-100 \mathrm{~nm}$ and lengths of $0.3-3 \mu \mathrm{m}$, while for the smaller Ln ions (Dy$\mathrm{Lu}$, and $\mathrm{Y}$ ), the lanthanide phosphates were obtained in the tetragonal phase and spherelike smaller morphologies which showed a reduced tendency to grow along a certain direction. Based on hydrothermal method but at slightly higher temperature and with different monomer sources, Zhang et al. ${ }^{10}$ have reported the fabrication of lanthanide orthophosphates of slightly different phase structures and morphologies. They fabricated the larger lanthanide ions (La-Gd) in orthophosphates of monoclinic structure and nanorod shapes with width of $10-100 \mathrm{~nm}$ and length of $1-10 \mu \mathrm{m}$. The smaller lanthanide ions (Dy-Lu and Y) were fabricated in the tetragonal phase spherelike morphologies with diameter of 20-150 nm, and only the $\mathrm{Tb}$ ions were obtained in the hydrated form $\mathrm{TbPO}_{4} \cdot 0.5 \mathrm{H}_{2} \mathrm{O}$ that adopted the hexagonal structure and nanorod morphologies with width $20-150 \mathrm{~nm}$ and length of 0.5-2 $\mu \mathrm{m}$. Lehmann et al. ${ }^{14}$ have reported the fabrication of lanthanide orthophosphate series employing the solution-precipitation method. In contrast to the previous results, they reported spherelike morphologies for the whole series with diameters in the range of 2.6-7 $\mathrm{nm}$. Additionally, the orthophosphates accommodating the intermediate lanthanide ions (Eu-Ho) adopted a mixed phase between that of monoclinic and tetragonal, and showed very small particle sizes with mean particle diameter of $2.6 \mathrm{~nm}$ that lead to a high surface-to-volume ratio.

In order to investigate the influence of crystal structure and morphology in the electronic properties of the lanthanide phosphates nanoparticles, we chose for investigation the one synthesized by the method of Lehmann et al. ${ }^{14}$ for the reason that these systems show a high dispersity. In this work, we present our experimental findings on the geometric and electronic structures of these systems. We deposited monolayer cluster films on crystalline silicon substrates and characterized them with scanning electron microcopy (SEM). We determined with X-ray powder diffraction the geometric structure of these systems. In addition, we determined the local electronic structure and the oxidation state of the lanthanide ions with near-edge X-ray absorption fine structure (NEXAFS) at the $M_{4,5}$-edge of each lanthanide. Finally, chemical bonding within the phosphate group in the nanoparticles and its interplay with the crystal phase and crystal dimension has been studied by oxygen $K$-edge NEXAFS.

\section{EXPERIMENT}

\section{A. Colloidal chemistry and synthesis}

Colloidal chemistry has been used extensively to synthesize monodisperse soluble particles of the nanometer scale. The working principle of this method is that in a supersaturated solid solution, where the system is kinetically over- driven by a high monomer concentration, diffusion effects bring about the formation of grains of a new phase. ${ }^{15}$ Further, the temperature controlled nucleation in the presence of coordinating surfactants follows controlled steady growth of the existing nuclei. ${ }^{16}$ The surfactants passivate the particles surface, i.e., the dangling bonds are terminated with an organic addend that reduces significantly the chemical reactivity of the clusters. Based on this method, ${ }^{14,3}$ the whole series of lanthanide orthophosphate nanoparticles has been synthesized. The particles surface is coordinated by amine chains that are responsible for their colloidal solubility and stability with respect to agglomeration. The crystallinity of the particles is investigated using the X-ray powder diffraction technique. The size distribution and the shape of the nanoparticles are examined by making use of the different methods: dynamic light scattering, X-ray diffraction (XRD), and transmission electron microscopy (TEM).

\section{B. Thin film preparation}

The lanthanide nanocrystals were dissolved in methanol solutions at $0.25 \%$ and $1 \%$ weight concentrations and then spin coated on silicon wafers. The substrates of $12 \mathrm{~mm}^{2}$ size and $525 \mu \mathrm{m}$ thickness were mirror-polished silicon single crystals of the (100) orientation. They were of $p$-type (doped with boron) with a resistivity of $\sim 5 \Omega \mathrm{cm}$. Prior to the spincoating procedure, a surface cleaning and passivation procedure was applied to the silicon wafers.

The silicon substrates were cleaned ultrasonically in acetone, in isopropanol, and then in de-ionized water for $10 \mathrm{~min}$, respectively. Afterward, they were chemically etched in solution of 10\% hydrofluoric acids (HF):50\% ethanol:40\% water for $10 \mathrm{~min}$, a recipe given by Ref. 17. The chemical HF etching of silicon wafers removes the surface oxide and terminates the silicon surface with atomic hydrogen. The hydrogen termination retards the silicon surface oxidation and protects the surface from chemical attack. In order to remove the physisorbed chemicals on the surface such as $\mathrm{SiF}_{4}, \mathrm{NH}_{4} \mathrm{OH}, \mathrm{HF}$, and other molecules, the wafers were rinsed briefly with de-ionized water ${ }^{18}$ subsequently after the chemical etching. Ultimately, a drop of lanthanide phosphate solution was spin coated on the cleaned and hydrogen-passivated surface for $1 \mathrm{~s}$ at $1000 \mathrm{rpm}, 11 \mathrm{~s}$ at $4000 \mathrm{rpm}$, and $19 \mathrm{~s}$ at $8000 \mathrm{rpm}$. The morphology of the thin film was investigated by SEM which showed a uniform distribution of the nanocrystals on the silicon surface. In the case of $0.25 \%$ concentration solutions, the surface coverage was less than one monolayer for all the lanthanide nanoparticles where the particle-packing ratio differed slightly from one lanthanide ion to the other. In Fig. 1, we show as an example the SEM images of the $\mathrm{ErPO}_{4}$ and $\mathrm{YbPO}_{4}$ nanoparticles of $0.25 \%$ concentration solution.

\section{X-ray spectroscopy and diffraction}

X-ray spectroscopy experiments were performed at the synchrotron radiation facilities Bessy II in Berlin and Hasylab in Hamburg at the beamlines U41 PGM and BW3. The spectroscopic investigations were performed on the Hamburg 


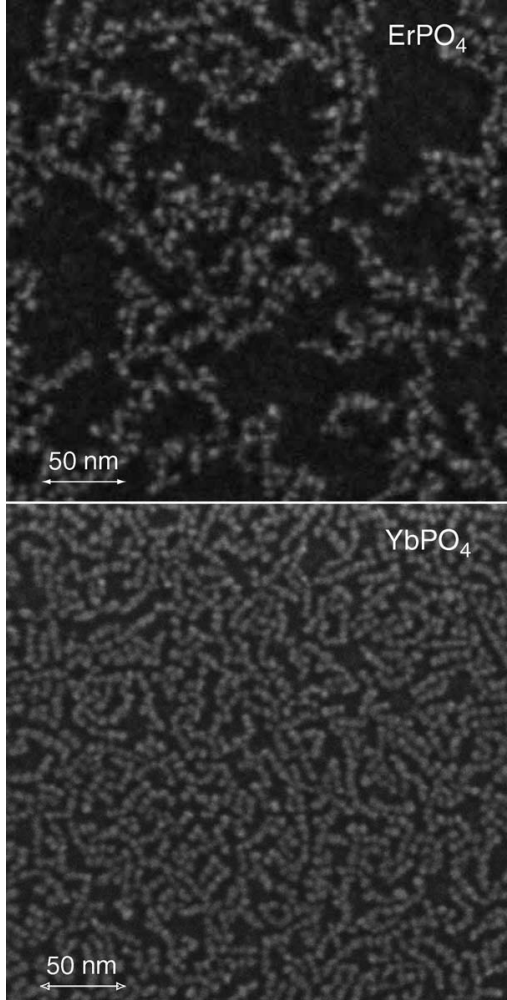

FIG. 1. Scanning electron micrographs: top panel of $\mathrm{ErPO}_{4}(300$ $\left.\times 300 \mathrm{~nm}^{2}\right)$ and bottom panel of $\mathrm{YbPO}_{4}\left(294 \times 294 \mathrm{~nm}^{2}\right)$ submonolayers spin coated on cleaned and hydrogen passivated silicon (100) surfaces. They show a uniform distribution of the nanoparticles at slightly different particlepacking ratio for $\mathrm{Er}$ and $\mathrm{Yb}$

inelastic X-ray scattering system that is equipped with a load-lock chamber, the preparation chamber and the spectroscopy chamber. The samples were brought into the loadlock chamber and were pumped for few minutes at a pressure of $1 \times 10^{-7}$ mbar; then, they were transferred through a transfer system into the UHV spectroscopy chamber equipped with a hemispherical photoelectron analyzer and a soft X-ray emission spectrometer. The synchrotron beam hit the sample under an angle of $7^{\circ}$ with respect to the surface plane. The photons were linearly polarized with polarization vector parallel to the sample surface. The resolving power $E / \Delta E$ of the photon beam was 2400 at the BW3 beamline for the oxygen $K$-edge and 3500 at the U41 PGM beamline for the $M_{4,5}$-edges of the lanthanides. The NEXAFS spectra were measured in the total electron yield mode by monitoring the sample current and in the constant emission energy mode by monitoring the Auger lines at a fixed energy window with the electron analyzer. The electron analyzer was collecting the electrons from the sample at the magic angle with respect to the linear polarization of the incident radiation.

X-ray diffraction experiments were performed at B2 beamline at the Hasylab synchrotron radiation facility in Hamburg equipped with a powder diffractometer and a image plate detector. ${ }^{19}$ The diffraction patterns of powder nanoparticles were collected from flat-sample transmission geometry with x-ray photons of energy $26.26 \mathrm{keV}$ (wavelength $\lambda=0.472 \AA$ ).

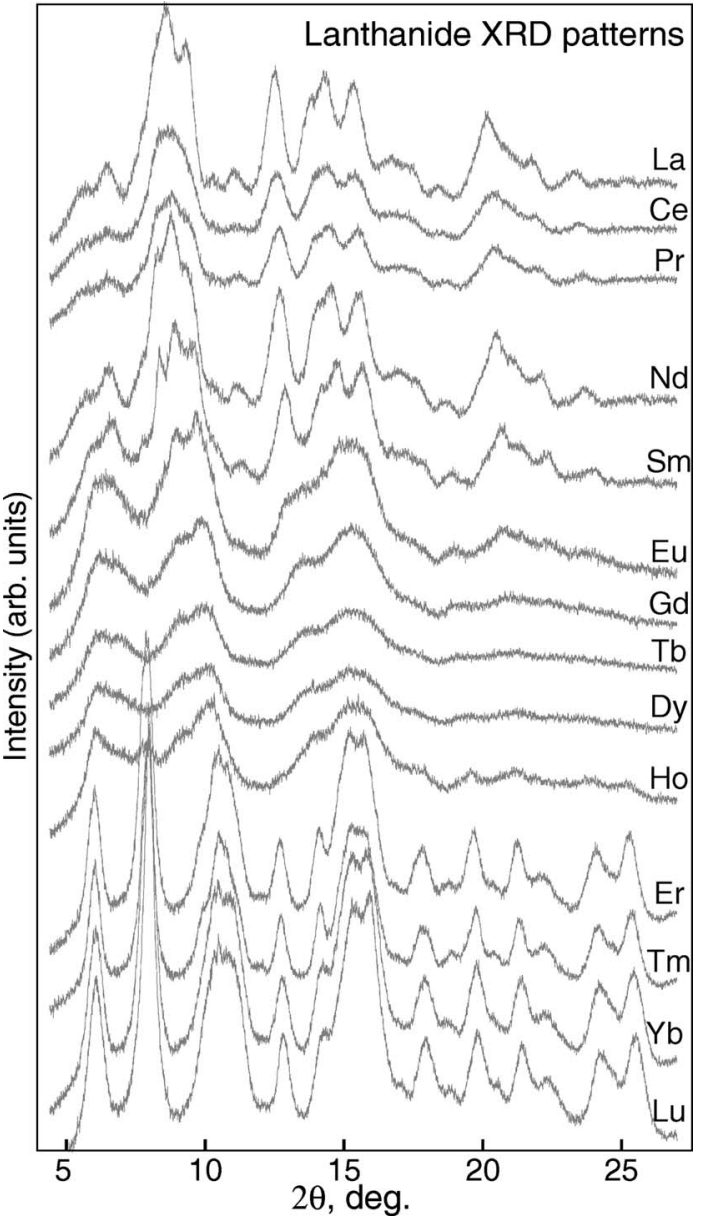

FIG. 2. X-ray powder diffraction patterns of lanthanide phosphate nanoparticles. Broad diffraction peaks indicate the small crystal size of 2-5 nm. La-Sm crystals are in monoclinic phase, Er-Lu crystals are in tetragonal phase, and Eu-Ho crystals are in mixed phase between monoclinic and tetragonal phase and are even smaller in size as seen from the peak width.

\section{RESULTS AND DISCUSSION}

\section{A. Long-range order in the crystallites}

The crystal structure of lanthanide orthophosphate nanoparticles has been identified by X-ray powder diffraction analysis. In Fig. 2, we show their diffractograms. All diffraction patterns are characterized by considerably broad reflection peaks owing to the small crystallite size of the nanoparticles. In the case of intermediate lanthanide ions (Eu, Gd, $\mathrm{Tb}$, Dy, Ho), the diffraction patterns are extremely broad, indicating a smaller crystallite size. The analysis of the diffraction patterns was done by full-profile Rietveld refinements ${ }^{20}$ that revealed the structures to be isotropic with no preferred direction. The mean particle size was roughly determined from the broadening of the reflection peaks using the Sherrer formula. ${ }^{21,22}$ The calculated diffraction patterns of the nanoparticles using the Rietveld structural refinement showed that the larger lanthanide ions (La, Ce, Pr, Nd, Sm) adopted the same monoclinic phase of monazite as their respective bulk crystals and the smaller ones (Er, Tm, Yb) adopted the same tetragonal structure of xenotime as their respective bulk crystals. In Fig. 3, we have shown the crystallographic calculations compared with the measured diffraction patterns for $\mathrm{LaPO}_{4}$ and $\mathrm{LuPO}_{4}$ nanoparticles. An 

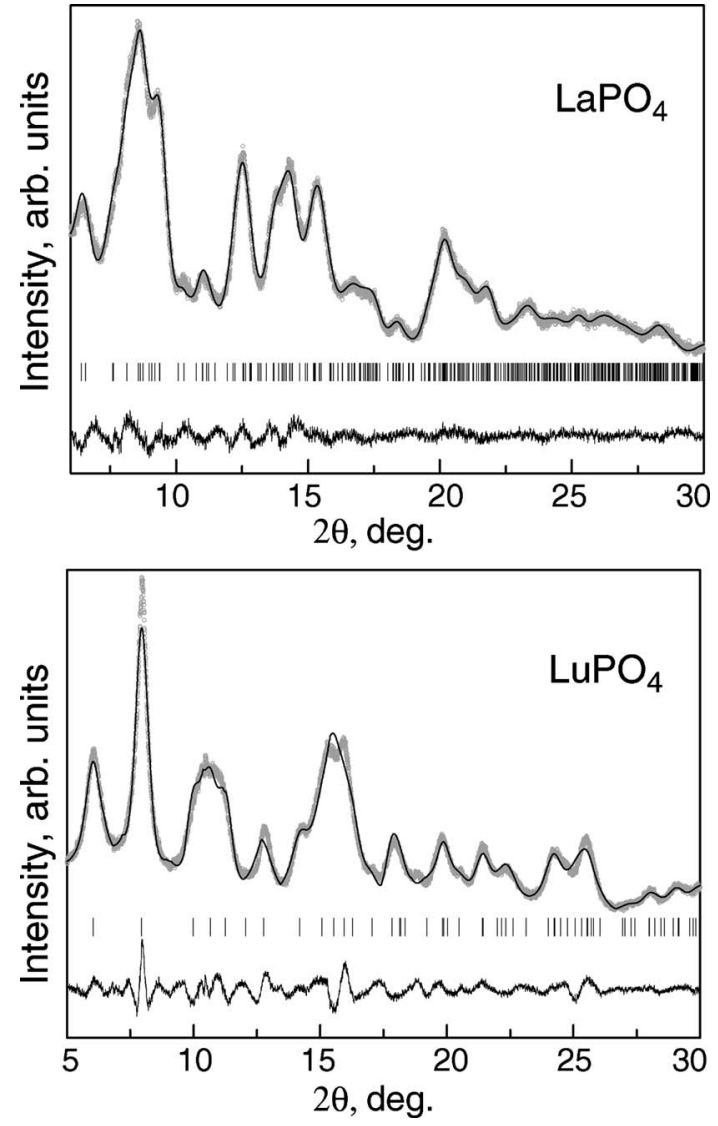

FIG. 3. Measured and calculated powder diffraction patterns for $\mathrm{LaPO}_{4}$ and $\mathrm{LuPO}_{4}$ nanoparticles. Crystallographic calculations were based on the monazite and xenotime structures (Ref. 23) for $\mathrm{LaPO}_{4}$ and $\mathrm{LuPO}_{4}$, respectively. Experimental data (circles) and calculated profiles (solid line through the circles) are presented together with the calculated Bragg positions (vertical ticks). The difference curve between the measured and calculated profiles is presented as a solid line below.

exception are the lanthanide nanoparticles from the middle of the series (Eu, Gd, Tb, Dy, Ho). In accordance with the previously reported results, ${ }^{14}$ these nanoparticles do not show the crystal phase of the respective bulks. Their diffractograms exhibit a crystal phase that is neither monoclinic nor tetragonal, but a "mixed phase" of monoclinic and tetragonal. In addition, our measurements revealed a slight phase transition from monoclinic to tetragonal, where Eu showed a crystal phase more similar to monoclinic and Ho showed a crystal phase more similar to tetragonal.

At large scattering angles (above $20^{\circ}$ ), the diffraction patterns of the intermediate lanthanides are characterized by very broad peaks due to diffraction from atomic planes at small interatomic distances of 1.5-2 $\AA$. The blurred diffraction patterns are a fingerprint for a missing short-range order of the atomic arrangements in these nanoparticles.

Inquiring into the reasons of the blurred patterns, we analyzed the morphologies and sizes of the nanoparticles. Highly resolved TEM images and particle-mean size calculations from the diffraction peak broadening revealed the very small particles of spherical shapes. In Fig. 4, highresolution TEM images of $\mathrm{PrPO}_{4}$ and $\mathrm{YbPO}_{4}$ nanoparticles are shown. The particle size within the lanthanide series exhibited the same trend as the crystal phase. The larger lan-

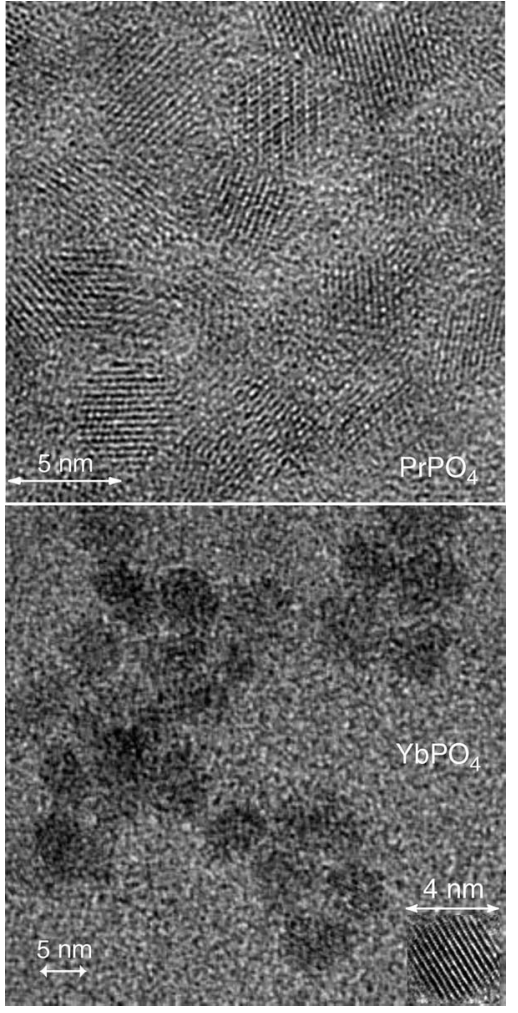

FIG. 4. High-resolution transmission electron micrographs: top panel of $\mathrm{PrPO}_{4}\left(22 \times 22 \mathrm{~nm}^{2}\right)$, bottom panel of $\mathrm{YbPO}_{4}\left(38 \times 38 \mathrm{~nm}^{2}\right)$ colloidal solutions. The inset shows an individual $\mathrm{YbPO}_{4}$ nanoparticle. The particles have spherelike morphology and sizes of $3-5 \mathrm{~nm}$.

thanide ions ( $\mathrm{La}, \mathrm{Ce}, \mathrm{Pr}, \mathrm{Nd}, \mathrm{Sm}$ ) and the smaller ones (Er, $\mathrm{Tm}, \mathrm{Yb}, \mathrm{Lu})$ showed to have a particle size of $\sim 3-5 \mathrm{~nm}$ diameter. The intermediate lanthanide ions ( $\mathrm{Eu}, \mathrm{Gd}, \mathrm{Tb}, \mathrm{Dy}$, Ho) showed very small particle sizes of about $\sim 2 \mathrm{~nm}$, which is accompanied with a high surface contribution in these particles. This trend of the crystal-phase and the crystal-size evolution through the lanthanide series was reported in Ref. 14 to be closely related to the decreasing effective cation radii with increasing the atomic number, known as "lanthanide contraction." For a detailed discussion, we are considering here the crystallographic studies of lanthanide orthophosphate bulk crystals. ${ }^{23} \mathrm{Ni}$ et al. have shown that distinct similarities exist between the monoclinic and tetragonal structures. Both atomic arrangements are based on [001] chains of intervening phosphate tetrahedra and lanthanide (Ln) polyhedra. As the structure "transforms" from monoclinic to tetragonal, the lanthanide polyhedron transforms from $\mathrm{LnO}_{9}$ to $\mathrm{LnO}_{8}$. Projected along the [001] direction, the tetrahedra-polyhedra chains exist in the (100) planes, with two planes per unit cell in both structures. In the monoclinic phase, the planes are offset by $2.2 \AA$ along [010] relative to those in the tetragonal phase, in order to accommodate the larger lanthanide atoms. The shift of the planes in the monoclinic phase allows the $\mathrm{Ln}$ atom to bond to an additional oxygen atom to complete the $\mathrm{LnPO}_{9}$ polyhedron. Additionally, $\mathrm{Ni}$ et al. showed that the unit cell of $\mathrm{TbPO}_{4}$ tetragonal is larger than that of $\mathrm{GdPO}_{4}$ monoclinic, despite the fact that $\mathrm{Tb}$ is smaller than Gd. This fact demonstrates that the void space in the tetragonal phase is larger compared to that in the 
monoclinic phase, thus, creating an energetically unfavorable situation. Thus, as the intermediate lanthanide particles have a very small size of $\leqslant 3 \mathrm{~nm}$, which corresponds to a surface to volume contribution of $\sim 70 \%$, a rearrangement of the atomic positions occurs so that the total energy of the system is minimized. Furthermore, because the lattice energies of the monoclinic and tetragonal phases close to the phase transition point are very similar, the atoms may occupy surface sites which are in between the monoclinic and tetragonal phases. This leads to a missing short-range order of the atomic arrangements that is fingerprinted in the very broad diffraction peaks. In the last section, we will discuss further the influence of the geometric structure and the mixed phase in the electronic structure of the lanthanide orthophosphate nanoparticles.

\section{B. Local structural geometry of the crystallites 1. $4 f$ occupancy of the lanthanide ions}

$M_{4,5}$-edge x-ray absorption spectroscopy applied on lanthanide ions is reported to be a useful method for studying the ground state $4 f$ electron occupancy and $4 f$ hybridization state, thus, revealing the (mean) valence state of the lanthanide ion. ${ }^{24}$ Therefore, we have measured the $M_{4,5}$-edge absorption spectra of lanthanide ions by monitoring the lanthanide MNN Auger lines in a fixed energy window. The results are shown in Fig. 5. The absorption spectra originate from the $3 d^{10} 4 f^{n} \rightarrow 3 d^{9} 4 f^{n+1}$ lanthanide transitions. Due to sufficiently deep $3 d$ shell, the spin-orbit interaction of the $3 d^{9}$ hole in the final state is much larger than the $3 d^{9} 4 f^{n+1}$ exchange interaction. Thus, the final states are split into two group of lines: $3 d_{5 / 2}\left(M_{5}\right)$ and $3 d_{3 / 2}\left(M_{4}\right)$. The $4 f$ electrons in the lanthanides are highly localized (lanthanide contraction) and are shielded from the interatomic interactions by the filled $5 s$ and $5 p$ outer electronic shells. In the presence of the core hole potential, the $4 f$ orbitals become stronger localized through the centrifugal barrier. As a consequence, the contribution from the higher empty $n f$ states $(n \geqslant 5)$ in the absorption spectra is suppressed, while the contribution from the higher $n p$ empty states is weak due to the low density of these states below the conduction band. ${ }^{25}$ Thus, the $M_{4,5}$-edge absorption spectra are due to $3 d^{10} 4 f^{n} \rightarrow 3 d^{9} 4 f^{n+1}$ electronic transitions. Each individual $M_{4}$ and $M_{5}$ absorption line shows a considerable multiplet structure. This fine structure is governed by the Coulomb and exchange interaction between the $3 d^{9}$ core hole and the $4 f$ electrons (which is strong due to the strong $4 f$ shell localization) and the spinorbit coupling of the core hole and $4 f$ electrons $(j-j$ coupling), i.e., the fine structure is due to the intermediate coupling of the $3 d^{9}$ hole and $4 f$ electrons in the lanthanide ions. The fine structure depends on the number of electrons in the $4 f$ shell because the spin-orbit interaction between the $4 f$ electrons and the exchange interaction between the $3 d$ hole and $4 f$ electrons will vary as a function of the number of electrons in the $4 f$ shell. This will lead to a different fine structure and different intensity ratios between the multiplets. Thus, the fine structure pattern of the $M_{4,5}$-edge absorption spectra is a trace of the electronic configuration of the $4 f$ subshell and it reveals the (mean) valence state of the lan-

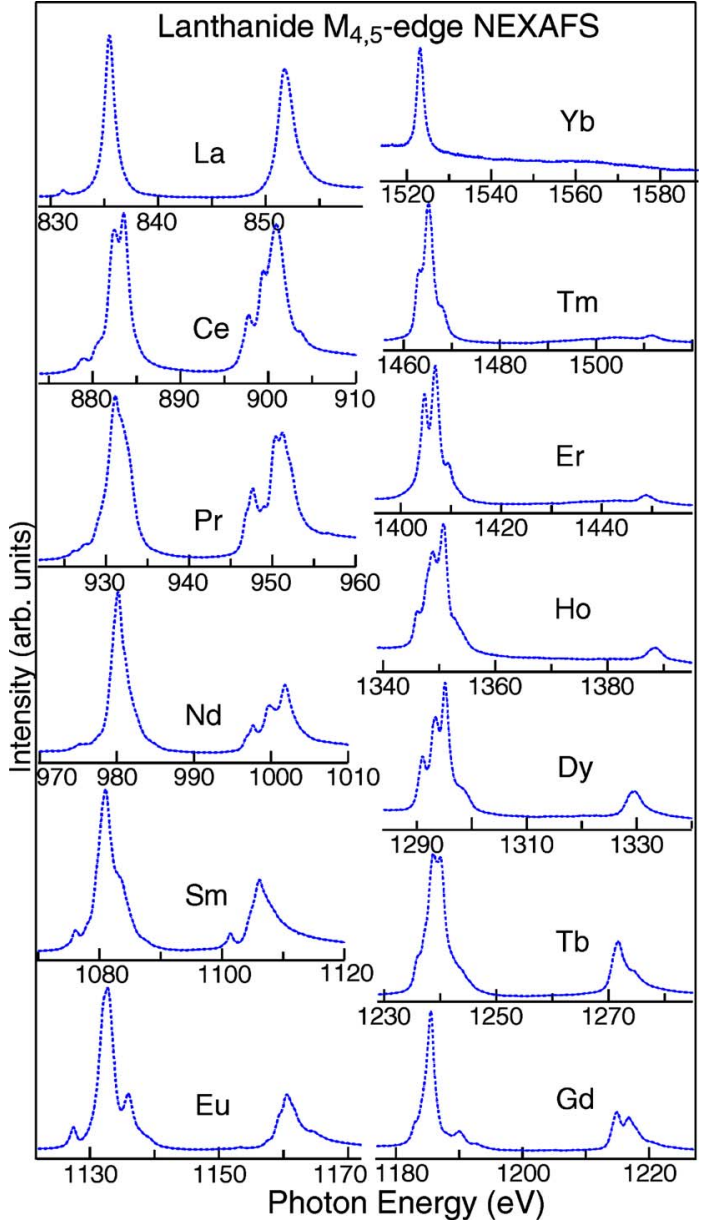

FIG. 5. (Color online) $M_{4,5}$-edge NEXAFS spectra of lanthanide ions measured by monitoring MNN Auger lines. Comparison of the characteristic multiplet structure of the spectra to the calculated one by Thole et al. (Ref. 25) reveal that all rare earth ions are in a triply ionized ground state.

thanide ion. We have compared the fine structure of our lanthanide X-ray absorption spectra with the one reported by Thole et al. ${ }^{25}$ They had performed Cowan's atomic multiplet Hartree-Fock calculations with relativistic corrections ${ }^{26}$ in the intermediate coupling, i.e., including all the states of the $4 f^{n}$ configuration for the ground state and $3 d^{9} 4 f^{n+1}$ final state, for all the lanthanide ions and for all ionization states known in the solid state. The comparisons show that all lanthanide ions in the orthophosphate nanoparticles are in the triply ionized ground state, establishing a $4 f$ occupancy in the ground state of $4 f^{0}$ for La until $4 f^{13}$ for Yb. In Figs. 6 and 7 , we have simulated the same calculations performed by Thole et al. for cerium and thulium ions. The calculated spectra are compared with our measured NEXAFS curves. In the case of cerium, an electronic ground state configuration of $4 f^{1}$ is revealed, while for thulium ions an electronic ground state configuration of $4 f^{12}$ is revealed. In both cases, theoretical results state a valence of III of cerium and thulium ions in the orthophosphate nanoparticles.

Because our $M_{4,5}$ NEXAFS spectra were recorded in the electron yield mode that allows observation of surface induced valence changes due to its moderate surface sensitivity, we state no surface induced valence changes in the nanoparticles due to their small crystal size. 


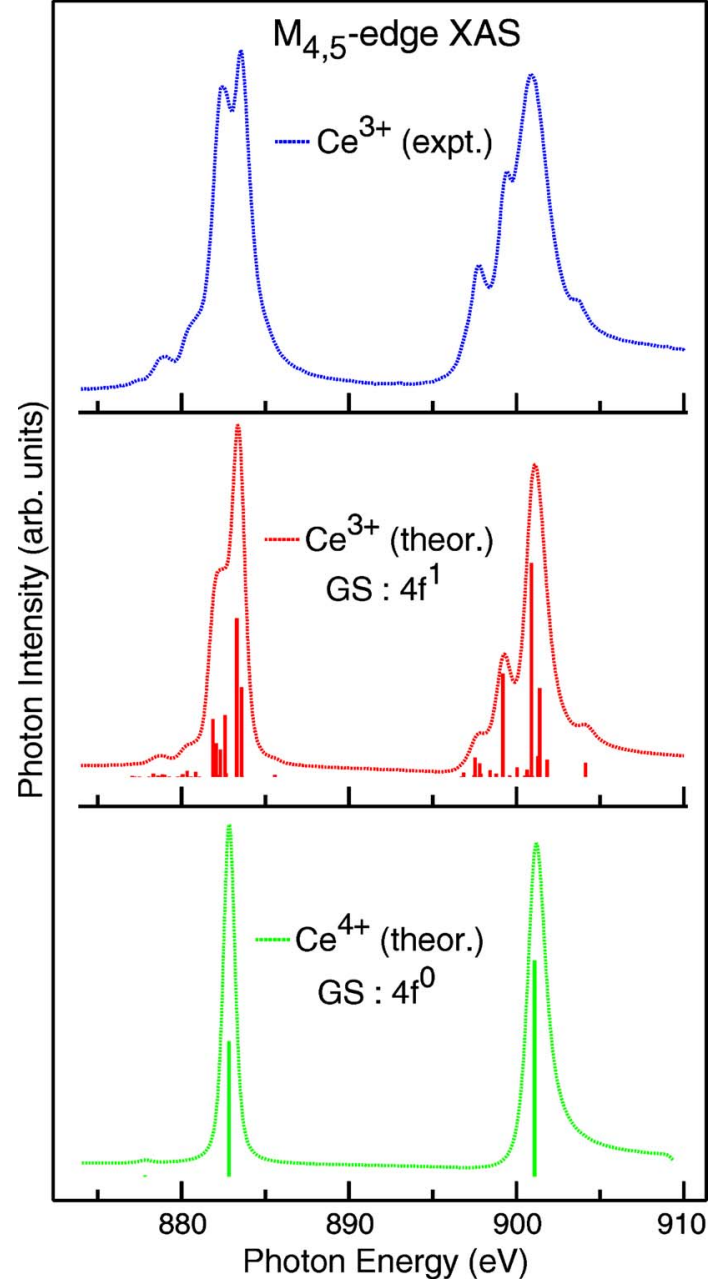

FIG. 6. (Color online) Experimental and theoretical spectra of cerium $M_{4,5}$-edge NEXAFS. Top panel: experimental NEXAFS curve. Bottom panels: atomic Hartree-Fock calculated spectra for ground state configuration $4 f^{1}$ and $4 f^{0}$, respectively.

The total integrated intensity of the $M_{4}$ and $M_{5}$ absorption lines decreases going from light lanthanides toward the heavier ones because the summed cross section of the two spin-orbit absorption bands is proportional to the number of valence holes ${ }^{27}$ and this number reduces going from lighter to the heavier lanthanides. However, the branching ratio of the two spin-orbit split lines $I\left(M_{5}\right) / I\left(M_{4}\right)+I\left(M_{5}\right)$ it is not given by its statistical value $6 / 4+6=0.4$. There are two effects that cause the change in the branching ratio. ${ }^{28}$ The first effect, is the presence of spin-orbit coupling in the initial state (due to the strong $4 f$ localization), that splits the $4 f$ valence electrons into bands of different values of the total atomic angular momentum quantum number $J(j-j$ coupling limit), and further the $J$ selection rules set preferences on the transitions to the two different manifolds. For this reason, $\mathrm{Yb}^{3+}$ absorption spectrum shows only one line, which is due to the fact that the only empty hole in the ground state is in the ${ }^{2} F_{7 / 2}$ state so that only the $3 d_{5 / 2} \rightarrow 4 f_{7 / 2}$ transition is allowed. The second effect is the presence of the $3 d$ hole- $4 f$ electron electrostatic interactions ( $L-S$ coupling) in the final state that couple the core hole to the valence holes differently

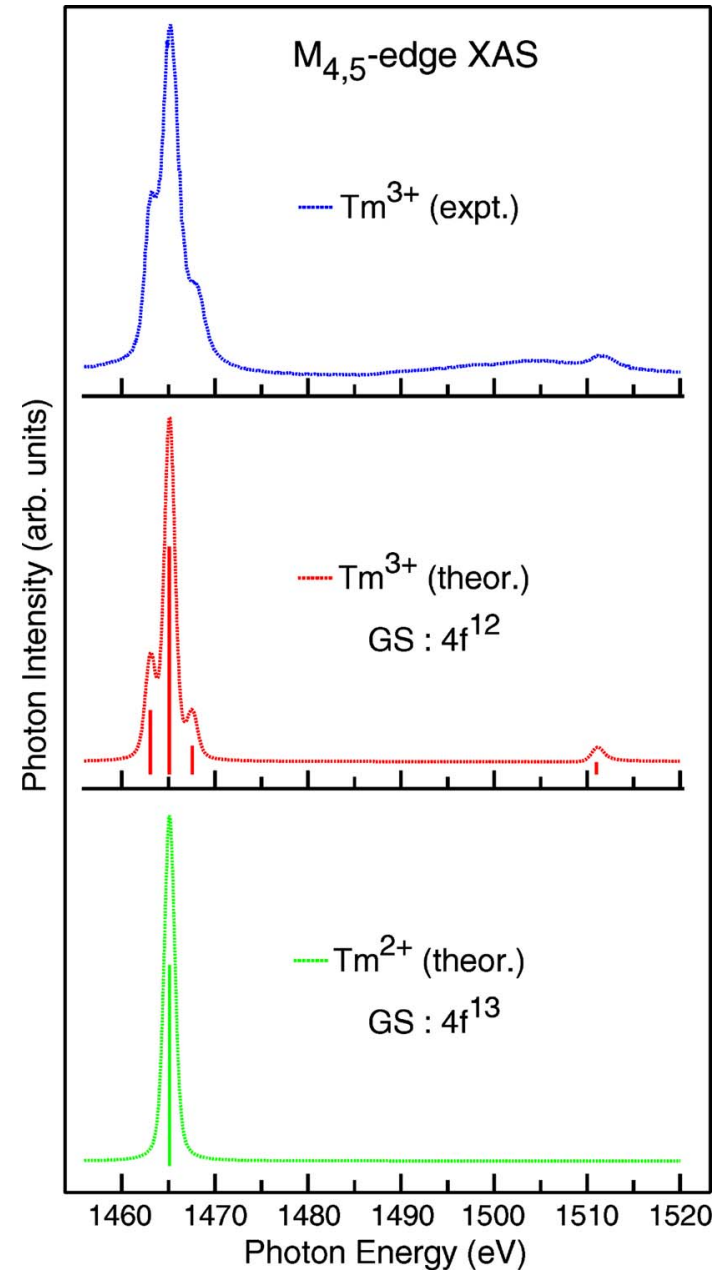

FIG. 7. (Color online) Experimental and theoretical spectra of thulium $M_{4,5}$-edge NEXAFS. Top panel: experimental NEXAFS curve. Bottom panels: atomic Hartree-Fock calculated spectra for ground state configuration $4 f^{12}$ and $4 f^{13}$, respectively.

for the two manifolds. ${ }^{28}$ (In the $L-S$ coupling limit, the spin selection rule will play a role in the transitions to the two different manifolds.)

Apart of the different branching ratio between the $M_{4}$ and $M_{5}$ absorption lines, also a lifetime broadening of the $M_{4}$ absorption line in comparison to the $M_{5}$ line is present, which is due to the Coster-Kronig decay. The $M_{4}$ XAS line is considered to be a superposition of the $3 d_{3 / 2} \rightarrow 4 f$ transitions and of the weak and continuous XAS due to the $3 d_{5 / 2}$ $\rightarrow$ to conduction band excitations, and the two processes are coupled each other by the Coster-Kronig transitions. The asymmetrical shape of the $M_{4}$ absorption line is due to the Fano resonance. ${ }^{29}$

\section{Lanthanide-oxygen covalency within the series}

In the following section, we will discuss the unoccupied valence electronic structure of the nanoparticles and examine its interplay with the particle's crystal size and crystal phase. For this purpose, the oxygen $K$-edge NEXAFS of all lanthanide nanoparticles is investigated, which is sensitive to the geometrical arrangement in the vicinity of the oxygen atom, and it gives information on the chemical bonding and the effective charge density around the oxygen atom. The 


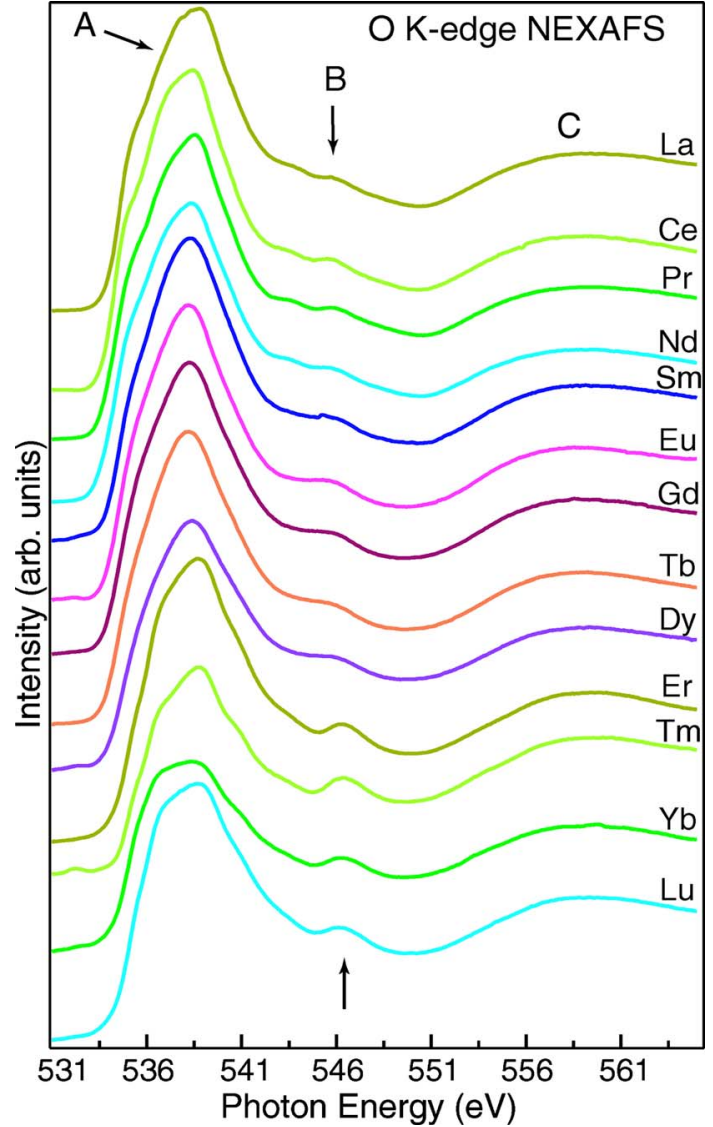

FIG. 8. (Color online) Oxygen $K$-edge NEXAFS spectra of lanthanide phosphate nanoparticles. Peaks A and B are due to Ln-O covalent bonding. The difference in their spectral shapes across the series is due to different geometric structures, coordination numbers, and particle morphologies. Peak B shifts toward higher energies and strengthens across the series indicating stronger covalency.

oxygen $K$-edge NEXAFS spectra of the whole lanthanide phosphate series (with exception of Ho) are shown in Fig. 8. The spectra are corrected for the background signal by subtracting the clean silicon substrate spectrum. NEXAFS signal correction in the case of $\mathrm{EuPO}_{4}$ sample is shown in Fig. 9. After background subtraction, all the spectra are normalized to the intensity at about $40 \mathrm{eV}$ above the absorption edge. The spectra consist of several peaks: the strong and broad peak located at $538 \mathrm{eV}$ denoted as $\mathrm{A}$, followed by the weak feature as $\mathrm{B}$, and the very broad shape resonance peak as C.

In order to discuss the origin of the different spectral features, we need to inquire into the band structure of lanthanide phosphates. In both monoclinic and tetragonal structures, the oxygen ions coordinate to two lanthanide ions and one phosphor ion. The oxygen-phosphor bonding is reported to be strongly covalent in the $\mathrm{PO}_{4}$ units and their conduction band consists of broad $\mathrm{O} 2 p$ projected density of states hybridized with $\mathrm{P}-3 p$ projected density of states. ${ }^{30-32}$

In the case of the lanthanides, the $4 f$ orbitals are strongly localized inside the atom and surrounded by the filled $5 s$ and $5 p$ orbitals that have larger radial extension. As a consequence, the $4 f$ orbitals do not take part in the bonding. Thus, the lowest unoccupied density of states in the lanthanide ions consist of $5 d$ character with $6 s$ and $6 p$ contribution. ${ }^{25}$ Hence, the strong broad peak A can be assigned to transitions from

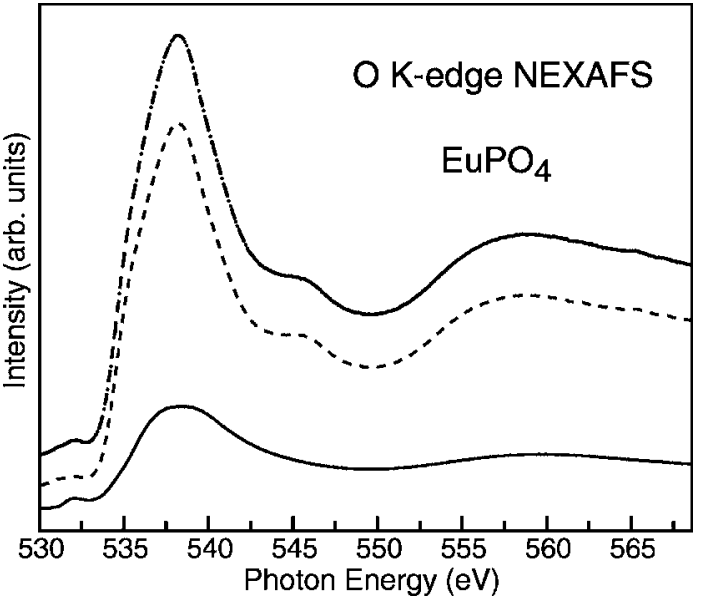

FIG. 9. O $K$-edge NEXAFS spectra of $\mathrm{Si}$ substrate-the solid line, $\mathrm{EuPO}_{4}$ nanoparticles on the Si substrate-the dashed circle line, and the $\mathrm{EuPO}_{4}$ nanoparticles subtracted the Si substrate signal-the dotted line.

$\mathrm{O} 1 s$ to empty $\mathrm{O} 2 p$ projected density of states hybridized with Ln $5 d$ and $\mathrm{P} 3 p$ projected density of states. Based on molecular-orbital theory, Grioni et al..$^{33}$ and de Groot et al. ${ }^{34}$ calculated the $\mathrm{O} 2 p$ orbitals to be spread over a high energy range above the Fermi level (up to $20 \mathrm{eV}$ ) in the transition metal oxides. They concluded that the pre-edge absorption peak is due to the hybridization of the antibonding $2 p$ orbitals with metal $3 d$ orbitals, and the absorption features located $5-10 \mathrm{eV}$ above the oxygen threshold have $2 p$ character hybridized with metal $4 s$ and $4 p$ orbitals. Proceeding under this assumption, we attribute the next peak B to the hybridization of $\mathrm{O} 2 p$ density of states with the upper-lying unoccupied density of states of Ln and P. In the case of lanthanide ions, the next upper-lying unoccupied density of states are $6 s$ and $6 p$ states. $^{25,35}$ Thus, the spectral feature B in the NEXAFS spectra is due to $\mathrm{O} 1 s$ to $2 p$ projected density of states hybridized with the Ln $6 s$ and $6 p$ and P $3 p$ density of states.

The same conclusions on the oxygen peak assignment were reached by Wu et al. based on ab initio one-electron full multiple-scattering (MS) calculations, i.e., considering the scattering of the outgoing photoelectron wave from the charge density close to the photoabsorber oxygen ion. They have performed calculations of the $\mathrm{O} K$-edge NEXAFS spectra of $\mathrm{Ba}_{2} \mathrm{TiO}_{4}$ (Ref. 35) that has monoclinic symmetry and of rutile $\mathrm{TiO}_{2}$ (Ref. 36) crystal that has a tetragonal symmetry. In the following, we would like to discuss the spectral shape variation of peaks A and B within the lanthanide series. The spectral shape difference of peak A in the monoclinic and tetragonal nanoparticles can be attributed to their different crystal structures ${ }^{34}$ and coordination numbers. Bearing in mind the geometric structure discussion of the previous section, in the monoclinic structure, one lanthanide ion is coordinated to nine oxygen ions, while in the tetragonal structure, it is coordinated to eight ions. Due to the less regular $\mathrm{LnO}_{9}$ polyhedra, the oxygen atoms accommodate in four different sites and show nine unique $\mathrm{Ln}-\mathrm{O}$ atomic distances in the monoclinic structure compared to only one oxygen site and four unique $\mathrm{Ln}-\mathrm{O}$ atomic distances in the tetragonal structure. Exact assignment of each subfeature would require 
detailed band structure calculations and is out of the scope of this paper.

The spectral feature $\mathrm{B}$ becomes more intense and locates at relatively higher energies going from $\mathrm{LaPO}_{4}$ nanoparticles of the monoclinic phase toward the $\mathrm{LuPO}_{4}$ nanoparticles. These facts reveal that the hybridization of $\mathrm{O} 2 p$ unoccupied density of states with Ln $6 s$ and $6 p$ unoccupied density of states is stronger in the tetragonal phase, thus, displaying stronger covalency between oxygen and lanthanide atoms in the tetragonal phase. Indeed, covalency reduces the number of filled states with $\mathrm{O} 2 p$ character so that the strength of the oxygen $K$-edge signal in the NEXAFS spectra is related to the degree of covalency. This conclusion is in agreement with the crystallographic data ${ }^{23}$ stating that the interatomic distance between lanthanide and oxygen atoms decreases going from monoclinic to tetragonal phase.

Finally, we want to discuss the width evolution of the spectral features A and B across the series. It can be seen from Fig. 8 that the spectral shapes of features A and B broaden at the intermediate lanthanide ions. For explaining this behavior, we recall here the discussion about the particles size and their short-range order of the previous section. We mentioned that the particles of the monoclinic and tetragonal phase exhibit a mean-crystal size of $3-5 \mathrm{~nm}$, with a surface to bulk contribution of $\sim 50 \%$. This causes a degree of disorder in the nanoparticle structure and respectively in the oxygen and lanthanide sites that results in broadening of the spectral shapes. The lanthanide nanoparticles from the middle of the series crystallize in even smaller size $(\sim 2 \mathrm{~nm})$ and have a more distorted geometry that results in the mixed phase of these nanoparticles. This increases the degree of disorder and, consequently, the number of oxygen and lanthanide sites in the nanoparticles and therefore the spectral shape of features A and B broadens further.

\section{CONCLUSIONS}

The evolution and interplay of the geometrical and electronic structures of lanthanide orthophosphate nanoparticles have been determined across the lanthanide series. The larger ( $\mathrm{La}, \mathrm{Ce}, \mathrm{Pr}, \mathrm{Nd}, \mathrm{Sm}$ ) and smaller (Er, Tm, Yb, Lu) lanthanide ions crystallize in the monoclinic and tetragonal phases, respectively, as their bulk crystals and exhibit particle sizes of $\sim 3-\sim 5 \mathrm{~nm}$. The intermediate lanthanide ions ( $\mathrm{Eu}, \mathrm{Gd}, \mathrm{Tb}$, Dy, Ho) show a very small particle size of $\sim 2 \mathrm{~nm}$ and crystallize in a mixed phase between monoclinic and tetragonal phases, induced by their large surface contribution and their ionic radii. The $M_{4,5}$-edge NEXAFS measurements established that all the lanthanide ions in the lanthanide phosphate nanocrystals are in a " $3+$ " configuration state. From the oxygen $K$-edge NEXAFS, we accessed the covalent bond involving the phosphate oxygen atoms toward the lanthanide ions. We tracked this covalency as a function of lanthanide ion, its coordination number to oxygen, and site symmetry. We showed that there is a stronger covalency between oxygen and lanthanide ions in the tetragonal phase compared to the monoclinic phase. The increased surface contribution and the missing of short-range order in the intermediate lan- thanide nanoparticles was accompanied by broad spectral shapes in oxygen $K$-edge NEXAFS and concomitant multiple oxygen and lanthanide sites.

\section{ACKNOWLEDGMENTS}

We owe special thanks to Frank de Groot for helpful discussions. We greatly acknowledge the contributions of Kerstin Rücker on synthesizing and characterizing the nanoparticles, of Andrea Kornowski on SEM investigations, and of Karsten Kömpe on TEM investigations of the nanostructures. We thank the scientific and technical staff of Bessy II and Hasylab for assistance.

This work was supported by the Graduiertenkolleg project: "Spektroskopie an lokalisierten atomaren Systemen, Felder und lokalisierte Atome-Atome und lokalisierte Felder" and the DFG Sonderforschungsbereich 508 "Quantenmaterialien—laterale Strukturen, hybride Systeme und Cluster."

${ }^{1}$ P. Moriarty, Rep. Prog. Phys. 64, 297 (2001).

${ }^{2}$ A. P. Alivisatos, Science 271, 933 (1996).

${ }^{3}$ K. Riwotzki, H. Meyssamy, A. Kornowski, and M. Haase, J. Phys. Chem. B 104, 2824 (2000)

${ }^{4}$ S. Heer, O. Lehmann, M. Haase, and H.-U. Güdel, Angew. Chem., Int. Ed. 42(27), 3179 (2003).

${ }^{5}$ G. A. Hebbink, J. W. Stouwdam, D. N. Reinhoudt, and F. C. van Veggel, Adv. Mater. (Weinheim, Ger.) 14, 1147 (2002).

${ }^{6}$ R. Yan, X. Sun, X. Wang, Q. Peng, and Y. Li, Chem.-Eur. J. 11, 2183 (2005).

${ }^{7}$ Y.-P. Fang, A.-W. Xu, R.-Q. Song, L.-P. You, H.-X. Zhang, J. C. Yu, and H.-Q. Liu, J. Am. Chem. Soc. 125, 16025 (2003).

${ }^{8}$ J. Nipko, C.-K. Loong, M. Loewenhaupt, M. Braden, W. Reichardt, and L. A. Boatner, Phys. Rev. B 56, 11584 (1997).

${ }^{9}$ X. Peng, L. Manna, W. Yang, J. Wickham, E. Scher, A. Kadavanich, and A. Alivisatos, Nature (London) 404, 59 (2000).

${ }^{10}$ Y.-W. Zhang, Z.-G. Yan, L.-P. You, R. Si, and C.-H. Yan, Eur. J. Inorg. Chem. 2003, 4099 (2003).

${ }^{11}$ G. Stryganyuk, D. Trots, A. Voloshinovski, T. Shalapska, V. Zakordonskiy, V. Vistovskyy, M. Pidzyrailo, and G. Zimmerer, J. Lumin. 128(3), 355 (2007).

${ }^{12}$ O. Lehman, K. Kömpe, and M. Haase, J. Am. Chem. Soc. 126, 14935 (2004).

${ }^{13}$ K. Kömpe, O. Lehmann, and M. Haase, Chem. Mater. 18, 4442 (2006).

${ }^{14}$ O. Lehmann, H. Meyssamy, K. Kömpe, H. Schnablegger, and M. Haase, J. Phys. Chem. B 107, 7449 (2003).

${ }^{15}$ I. Lifshitz and V. Slyozov, J. Phys. Chem. Solids 19, 35 (1961).

${ }^{16}$ C. Murray, D. Norris, and M. G. Bawendi, J. Am. Chem. Soc. 115, 8706 (1993).

${ }^{17}$ J. Thornton and R. Williams, Phys. Scr. 41, 1047 (1990).

${ }^{18}$ P. Dumas, Y. Chabal, and P. Jakob, Surf. Sci. 269/270, 867 (1992).

${ }^{19}$ M. Knapp, C. Baehtz, H. Ehrenberg, and H. Fuess, J. Synchrotron Radiat. 11, 328 (2004).

${ }^{20}$ T. Roisnel and J. Rodriquez-Carvajal, Mater. Sci. Forum 378/381, 118 (2001).

${ }^{21}$ P. Sherrer, Nachr. Ges. Wiss. Goettingen, Math.-Phys. Kl. 2, 96 (1918).

${ }^{22}$ S. Howard and K. Preston, Modern Powder Diffraction (Mineralogical Society of America, Washington, DC, 1989), Vol. 20.

${ }^{23}$ Y. Ni, J. M. Hughes, and A. N. Mariano, Am. Mineral. 80, 21 (1995).

${ }^{24}$ G. Kaindl, G. Kalkovski, W. D. Brewer, B. Perscheid, and F. Holtzberg, J. Appl. Phys. 55, 1910 (1984).

${ }^{25}$ B. T. Thole, G. van der Laan, J. C. Fuggle, G. A. Sawatzky, R. C. Karnatak, and J.-M. Esteva, Phys. Rev. B 32, 5107 (1985).

${ }^{26}$ R. D. Cowan, The Theory of Atomic Structure and Spectra (University of California Press, Berkeley, CA, 1981).

${ }^{27}$ B. T. Thole and G. van der Laan, Europhys. Lett. 4, 1083 (1987).

${ }^{28}$ B. T. Thole, Phys. Rev. B 38, 3158 (1988).

${ }^{29}$ U. Fano, Phys. Rev. 124, 1866 (1961).

${ }^{30} \mathrm{~S}$. Kucheyev, C. Bostedt, T. van Buuren, T. Willey, T. Land, L. Terminello, T. Felter, A. Hamza, and S. Demos, Phys. Rev. B 70, 245106 
(2004).

${ }^{31}$ Z. Lin, Z. Wang, C. Chen, and M.-H. Lee, J. Chem. Phys. 118, 2349 (2003).

${ }^{32}$ M. Willinger, D. Su, and R. Schlögl, Phys. Rev. B 71, 155118 (2005).

${ }^{33}$ M. Grioni, M. Czyzyk, F. de Groot, J. Fuggle, and B. Watts, Phys. Rev. B 39, 4886 (1989).
${ }^{34}$ F. de Groot, M. Grioni, J. Fuggle, J. Ghijsen, G. Sawatzky, and H. Petersen, Phys. Rev. B 40, 5715 (1989).

${ }^{35}$ Z. Wu, E. Paris, F. Langenhorst, and F. Seifert, J. Synchrotron Radiat. 9, 394 (2002).

${ }^{36}$ Z. Wu, S. Gota, F. Jollet, M. Pollak, M. Gautier-Soyer, and C. Natoli, Phys. Rev. B 55, 2570 (1997). 